\title{
THE TRACE FORMULA IN BANACH SPACES
}

\author{
W. B. JOHNSON AND A. SZANKOWSKI
}

\begin{abstract}
A classical result of Grothendieck and Lidskii says that the trace formula (that the trace of a nuclear operator is the sum of its eigenvalures provided the sequence of eigenvalues is absolutely summable) holds in Hilbert spaces. In 1988 Pisier proved that weak Hilbert spaces satisfy the trace formula. We exhibit a much larger class of Banach spaces, called $\Gamma$-spaces, that satisfy the trace formula. A natural class of asymptotically Hilbertian spaces, including some spaces that are $\ell_{2}$ sums of finite dimensional spaces, are $\Gamma$-spaces. One consequence is that the direct sum of two $\Gamma$-spaces need not be a $\Gamma$-space.
\end{abstract}

Dedicated to the memory of Joram Lindenstrauss

\section{INTRODUCTION}

Let $X$ be a Banach space. $L(X)$ denotes the space of bounded operators on the space $X$ while $F(X)$ denotes the finite rank operators in $L(X) . B_{X}$ denotes the unit ball of $X$. The identity operator on $X$ is written $I_{X}$.

For $x^{*} \in X^{*}, x \in X$ let $x^{*} \otimes x \in F(X)$ be defined by

$$
\left(x^{*} \otimes x\right)(y)=x^{*}(y) x .
$$

Every $T \in F(X)$ can be represented in the form

$$
T=\sum_{i=1}^{n} x_{i}^{*} \otimes x_{i} \text { with } x_{1}^{*}, \ldots, x_{n}^{*} \in X^{*}, x_{1}, \ldots, x_{n} \in X .
$$

By elementary algebra, the sum

$$
\operatorname{tr} T=\sum_{i=1}^{n} x_{i}^{*}\left(x_{i}\right)
$$

Date: March 1, 2013.

1991 Mathematics Subject Classification. Primary 46B20, 46B07; Secondary 46B99.

Key words and phrases. trace formula, almost Hilbert spaces, asymptotically Hilbertian spaces.

Johnson was supported in part by NSF DMS-1001321 and the U.S.-Israel Binational Science Foundation. 
is well defined, i.e. does not depend on the representation of $T$. A much deeper elementary fact is that for $T \in F(X)$ the trace formula:

$$
\operatorname{tr} T=\sum \lambda_{j}(T)
$$

holds. Here $\lambda_{1}(T), \lambda_{2}(T), \ldots$ are all the eigenvalues of $T$, with their multiplicities (we suppose that $X$ is a complex Banach space).

It is natural to seek generalizations of these facts to the infinite dimensional setting.

A $T \in B(X)$ is called nuclear if

$$
T=\sum_{i=1}^{\infty} x_{i}^{*} \otimes x_{i} \text { with } \sum\left\|x_{i}^{*}\right\|\left\|x_{i}\right\|<\infty .
$$

By $N(T)$ we denote the space of all nuclear operators on $X$. In $N(X)$ we define the following norm (called the nuclear norm):

$$
\|T\|_{\wedge}=\inf \left\{\sum\left\|x_{i}^{*}\right\|\left\|x_{i}\right\|: T=\sum_{i=1}^{\infty} x_{i}^{*} \otimes x_{i}\right\} .
$$

Grothendieck [4] (cf. [11, Theorem 1.a.4.]) discovered that if $X$ has the approximation property (AP), then for every $T \in N(X), \operatorname{tr} T=$ $\sum_{i=1}^{\infty} x_{i}^{*}\left(x_{i}\right)$ is well defined.

Suppose that $X$ is a complex Banach space with the AP. We ask whether

(L) the trace formula (1) holds for every $T \in N(X)$ which has absolutely summable eigenvalues $\lambda_{1}(T), \lambda_{2}(T), \ldots$

(this assumption is necessary, because for every $X$ not isomorphic to a Hilbert space there is a $T \in N(X)$ such that $\sum\left|\lambda_{j}(T)\right|=\infty$, by a result in [7]).

In [9] Lidskii proved that the answer to $(\mathrm{L})$ is positive if $X$ is a Hilbert space. As was pointed out by Pisier [15], Grothendieck was aware of this result somewhat earlier [5].

For general $X$, the answer is negative. Spaces which satisfy condition (L) will be called Lidskii spaces.

It turns out that Lidskii spaces are very close to Hilbert spaces. Let us say that $X$ is an almost Hilbert space if $X$ is of type $(2-\varepsilon)$ and of cotype $(2+\varepsilon)$ for every $\varepsilon>0$.

Recall the definitions of type and cotype. Let $\left(r_{i}\right)$ be a sequence of independent random variables taking the values 1 and -1 each with probability $1 / 2$. Given $n \in \mathbb{N}$ and $1 \leq p<\infty$, the type $p$ constant $T_{p}^{(n)}$ and the cotype $p$ constant $C_{p}^{(n)}$ are the smallest constants which satisfy the following inequalities for all $n$-tuples of vectors in $X$ : 


$$
\mathbb{E}\left\|\sum_{i=1}^{n} r_{i} y_{i}\right\|^{p} \leq T_{p}^{(n)}(X)^{p} \sum_{i=1}^{n}\left\|y_{i}\right\|^{p},
$$

respectively

$$
C_{p}^{(n)}(X)^{p} \mathbb{E}\left\|\sum_{i=1}^{n} r_{i} y_{i}\right\|^{p} \geq \sum_{i=1}^{n}\left\|y_{i}\right\|^{p} .
$$

$T_{p}(X):=\lim _{n \rightarrow \infty} T_{p}^{(n)}(X)$ and $C_{p}(X):=\lim _{n \rightarrow \infty} C_{p}^{(n)}(X)$ are the type $p$ and cotype $p$ constants for $X$. The space $X$ is said to be of type $p$; respectively, of cotype $p$, provided $T_{p}(X)<\infty$; respectively, $C_{p}(X)<$ $\infty$.

For technical reasons, we also consider a weakened version of (L) which we term (WL); namely, that every quasi-nilpotent nuclear operator on $X$ has trace zero. (The "technical reasons" are that in the unwritten paper [3] it is proved that (WL) implies (L) and we find it easier to verify that certain spaces satisfy (WL) rather than check that they satisfy $(\mathrm{L}))$.

The weak Lidskii property (WL) implies the following property, which in turn implies that a Banach space $X$ that satisfies (L) is an almost Hilbert space:

(HAP) $X$ has the hereditary approximation property; that is, all of its subspaces have the AP.

(See [8] and the references therein).

Until now, the only spaces known to satisfy (L) are the weak Hilbert spaces (cf. [15, Chpt. 12], [16]; "weak Hilbert" is defined in the next section). Pisier built a beautiful theory of weak Hilbert spaces and there are some important weak Hilbert spaces, such as the 2 convexification of Tsirelson's space; [15, Chpt. 13]. However, the weak Hilbert spaces are somewhat elusive and there are very few known examples of them; in particular, Hilbert spaces are the only classical Banach spaces that are weak Hilbert spaces.

In this paper we exhibit a much larger class of spaces which satisfy the condition (WL). Nevertheless, our approach is a direct outgrowth of Pisier's approach to weak Hilbert spaces. We show that a Banach space that satisfies a weakened version of one of Pisier's equivalent conditions for being a weak Hilbert spaces must be a weak Lidskii space. It is relatively easy to show that many non weak Hilbert spaces, including some classical spaces other than Hilbert spaces, satisfy this weakened condition. 


\section{2. $\Gamma$-SPACES}

For $\varphi=\left(\varphi_{1}, \ldots, \varphi_{n}\right) \in B_{X^{*}}^{n}$ and $x=\left(x_{1}, \ldots, x_{n}\right) \in B_{X}^{n}$, let

$$
G(\varphi, x)=\operatorname{det}\left[\left\langle\varphi_{i}, x_{j}\right\rangle\right]_{i, j=1}^{n} \text {. }
$$

Define

$$
\begin{gathered}
G_{n}(X)=\sup \left\{|G(\varphi, x)|: \varphi \in B_{X^{*}}^{n}, x \in B_{X}^{n}\right\} . \\
\Gamma_{n}(X)=G_{n}(X)^{\frac{1}{n}} .
\end{gathered}
$$

If $\operatorname{dim} E=n<\infty$ we denote

$$
G(E)=G_{n}(E), \Gamma(E)=\Gamma_{n}(E) .
$$

Let us observe that

$$
G(E) \geq 1 \text { for every } E \text {. }
$$

Indeed, let $\left\{x_{i}^{*}, x_{i}\right\}_{i=1}^{n}$ be an Auerbach system [11, Proposition 1.c.3] for $E$, i.e. $x_{i}^{*} \in B_{E^{*}}, x_{i} \in B_{E}$ and $x_{i}^{*}\left(x_{j}\right)=\delta_{i j}$. Then, clearly, $G\left(x_{1}^{*}, \ldots, x_{n}^{*} ; x_{1}, \ldots, x_{n}\right)=1$.

Define

$$
\Gamma_{\text {sup }}(X)=\sup \Gamma_{n}(X) \text { and } \Gamma_{\inf }(X)=\liminf \Gamma_{n}(X)
$$

Let us recall that a Banach space $X$ is a weak Hilbert (WH) space if

$$
\Gamma_{\sup }(X)<\infty \text {. }
$$

This is one of several equivalences to a Banach space $X$ being a WH space ([6, Theorem 15.1]).

We say that a Banach space $X$ is a $\Gamma$-space provided

$$
\Gamma_{\inf }(X)<\infty
$$

This is a substantial relaxation of the WH condition; nevertheless, as we show in this paper, the $\Gamma$-spaces still behave very much like $\mathrm{WH}$ spaces. In particular, they satisfy (L).

By $d_{n}(X)$ we denote the supremum over the $n$-dimensional subspaces $E$ of $X$ of the isomorphism constant from $E$ to $\ell_{2}^{n}$; that is, $d_{n}(X)=$ $\sup \left\{d\left(E, l_{2}^{n}\right): E \subset X, \operatorname{dim} E=n\right\}$. The isomorphism constant from $E$ to $F, d(E, F)$, is the infimum of $\|T\| \cdot\left\|T^{-1}\right\|$ as $T$ ranges over all isomorphisms from $E$ onto $F$.

A few elementary facts concerning $G_{n}(X)$ and $\Gamma_{n}(X)$ : 
Lemma 2.1. For any $X, Y, n$ we have

$$
\begin{gathered}
\Gamma_{n}(X) \leq d(X, Y) \Gamma_{n}(Y) \\
G_{1}(X) \leq G_{2}(X) \leq \ldots \\
G_{n}(X) \leq d_{n}(X) G_{n-1}(X) \leq n^{1 / 2} G_{n-1}(X) .
\end{gathered}
$$

Proof. (9) Let $T: X \rightarrow Y$ be an isomorphism. Given $\varphi=\left(\varphi_{1}, \ldots, \varphi_{n}\right) \in$ $B_{X^{*}}^{n}$ and $x=\left(x_{1}, \ldots, x_{n}\right) \in B_{X}^{n}$, let us define $\psi_{j}=\left(\left(T^{-1}\right)^{*}\right) \varphi_{j}, y_{j}=$ $T x_{j}$ for $j=1, \ldots, n$ and $\psi=\left(\psi_{1}, \ldots, \psi_{n}\right), y=\left(y_{1}, \ldots, y_{n}\right)$. We see that $G(\psi, y)=G(\varphi, x)$ and $\prod_{j=1}^{n}\left\|\psi_{j}\right\| \prod_{j=1}^{n}\left\|y_{j}\right\| \leq\left(\|T\|\left\|T^{-1}\right\|\right)^{n}$, which implies (9).

(10). Let $\varphi=\left(\varphi_{1}, \ldots, \varphi_{n}\right) \in B_{X^{*}}^{n}, x=\left(x_{1}, \ldots, x_{n}\right) \in B_{X}^{n}$. Let $\varphi_{n+1} \in X^{*}$ be such that $\left\|\varphi_{n+1}\right\|=1$ and $\operatorname{span}\left\{x_{1}, \ldots, x_{n}\right\} \subset \operatorname{ker} \varphi_{n+1}$, let $x_{n+1} \in B_{X}$ be such that $\varphi_{n+1}\left(x_{n+1}\right)=1$.

Then $G(\varphi, x)=G\left(\varphi_{1}, \ldots, \varphi_{n+1}, x_{1}, \ldots, x_{n+1}\right)$.

(11). Let us fix $x_{1}, \ldots, x_{n} \in B_{X}, \varphi_{1}, \ldots, \varphi_{n} \in B_{X^{*}}$. Let $E=$ span $\left\{x_{1}, \ldots, x_{n}\right\}$, set $\psi_{j}=\varphi_{j \mid E}, \psi_{j} \in E^{*}$. Let $\mid$ | be a euclidean norm in $E$ such that $d_{n}(X)^{-1}|x| \leq\|x\| \leq|x|$. Let us identify $\psi_{j}$ with the $\psi_{j} \in E$ such that $\left\langle\psi_{j}, x_{i}\right\rangle=\left(\psi_{j}, x_{i}\right)$ for $i=1, \ldots, n$, where $($,$) is the$ scalar product corresponding to the norm $\mid$ |.

For $y_{1}, \ldots, y_{k} \in E$ let $V\left(y_{1}, \ldots, y_{k}\right)$ denote the volume (induced by $()$,$) of the parallelpiped [0,1] y_{1}+\cdots+[0,1] y_{k}$.

We have $|G(\varphi, x)|=V\left(\psi_{1}, \ldots, \psi_{n}\right) V\left(x_{1}, \ldots, x_{n}\right)$. Since $\left|x_{n}\right| \leq d_{n}(X)$ and $\left|\psi_{n}\right| \leq 1$, we have $V\left(x_{1}, \ldots, x_{n}\right) \leq d_{n}(X) V\left(x_{1}, \ldots, x_{n-1}\right)$ and $V\left(\psi_{1}, \ldots, \psi_{n}\right) \leq V\left(\psi_{1}, \ldots, \psi_{n-1}\right)$. This gives the left inequality in (11). The right inequality in $(11)$, that $d_{n}(X) \leq n^{1 / 2}$, is a well known consequence of John's lemma about the maximal volume ellipsoid contained in the unit ball of a finite dimensional space [18, Theorem 6.30]).

Recall that a space $X$ is asymptotically Hilbertian provided there are subspaces $Y_{1}, Y_{2}, \cdots \subset X$ with $\operatorname{dim} X / Y_{n}<\infty$ and $\sup _{n} d_{n}\left(Y_{n}\right)<$ $\infty$. Observe that we obtain the same definition if this is replaced by the formally weaker condition $\liminf _{n} d_{n}\left(Y_{n}\right)<\infty$ (this is so because $d_{n}(X)$ is always a non-decreasing sequence).

If, additionally, such $Y_{n}$ can be chosen to be uniformly complemented in $X$, we say that $X$ is complementably asymptotically Hilbertian $(\mathrm{CAH})$.

We shall need the following fact

Proposition 2.1. Let $X$ be asymptotically Hilbertian. Then for every $\alpha>0, d_{n}(X)=O\left(n^{\alpha}\right)$. 
Proof. Recall [13, Lemma 13.4] that the numbers $T_{2}^{(n)}$ and $C_{2}^{(n)}$ are submultiplicative; that is,

$$
C_{2}^{(n m)}(Y) \leq C_{2}^{(n)}(Y) C_{2}^{(m)}(Y), \quad T_{2}^{(n)}(Y) \leq T_{2}^{(n)}(Y) T_{2}^{(m)}(Y) .
$$

In particular, for any natural number $\gamma$,

$$
C_{2}^{\left(n^{\gamma}\right)}(Y) \leq C_{2}^{(n)}(Y)^{\gamma}, \quad T_{2}^{\left(n^{\gamma}\right)}(Y) \leq T_{2}^{(n)}(Y)^{\gamma} .
$$

Consequently, using Kwapien's theorem [18, Theorem 13.15] in the first inequality below and the obvious inequality $\max \left\{C_{2}^{n}(Y), T_{2}^{n}(Y)\right\} \leq$ $d_{n}(Y)$ in the second, we get

$$
d_{n^{\gamma}}(Y) \leq\left(C_{2}^{(n)}(Y) T_{2}^{(n)}(Y)\right)^{\gamma} \leq d_{n}(Y)^{2 \gamma} .
$$

Let $\beta>0$ be such that for every $n$ there is a finite codimensional subspace $Y_{n} \subset X$ such that $d_{n}\left(Y_{n}\right) \leq \beta$. Let $m$ be such that $\beta^{2} \leq m^{\alpha}$. By $(14), d_{m \gamma}\left(Y_{m}\right) \leq \beta^{2 \gamma} \leq m^{\alpha \gamma}$. This clearly implies that $d_{n}\left(Y_{m}\right)=$ $O\left(n^{\alpha}\right)$. Since $\operatorname{dim} X / Y_{m}<\infty$, also $d_{n}(X)=O\left(n^{\alpha}\right)$.

Remark 2.1. One cannot replace $O\left(n^{\alpha}\right)$ by e.g. $O(\log n)$ : given $p_{n} \rightarrow 2$, consider $X=\left(\sum_{n=1}^{\infty} \oplus \ell_{p_{n}}^{n}\right)_{2}$. Then $d_{n}(X) \geq n^{\left|1 / 2-1 / p_{n}\right|}$.

In section 3 we prove that $\Gamma$-spaces are complementably asymptotically Hilbertian.

In section 4 we prove that $\Gamma$-spaces satisfy condition (WL) (and hence, by [3] also satisfy (L)).

In section 5 we exhibit a large class of asymptotically Hilbertian spaces which are $\Gamma$-spaces, thus obtaining many spaces which satisfy condition (WL).

\section{3. -SPACES ARE COMPLEMENTABLY ASYMPTOTICALLY HiLBERTIAN}

Theorem 3.1. $\Gamma$-spaces are complementably asymptotically Hilbertian.

Let $X$ be a Banach space and let $E$ be a finite dimensional space. We will say that $E$ is infinitely reproducible in $X$ if for every $\varepsilon>0$ there are $E_{1}, E_{2}, \ldots \subset X$ so that $E_{1} \oplus E_{2} \oplus \ldots$ is a $(1+\varepsilon)$ - Schauder decomposition

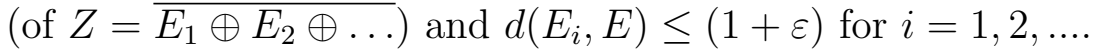

Proposition 3.1. Let $Y$ be an ultraproduct of the form $Y=\prod Y_{n} / \mathcal{U}$ where $\mathcal{U}$ is a free ultrafilter on $\mathbb{N}$ and $Y_{n}$ is a decreasing sequence of finite codimensional subspaces of $X$ with $\bigcup_{n} Y_{n}^{\perp}$ norm determining for $X$. Then every finite dimensional subspace of $Y$ is infinitely reproducible in $X$. 
Proof. Let $E \subset Y$ be finite dimensional. There exist $F_{n} \subset Y_{n}$ such that $\lim _{n \in \mathcal{U}} d\left(F_{n}, E\right)=1$. If $F$ is a finite dimensional subspace of $X$ that is $(1+\varepsilon)$-normed by $Y_{n}^{\perp}$, then the natural projection from $F+Y_{n}$ onto $F$ has norm at most $(1+\varepsilon)$. Consequently, we can extract from the sequence $F_{1}, F_{2}, \ldots$ a subsequence $E_{1}, E_{2}, \ldots$ such that, on one hand, $E_{1} \oplus E_{2} \oplus \ldots$ is a $(1+\varepsilon)$ - Schauder decomposition and, on the other hand, $d\left(E_{i}, E\right) \leq(1+\varepsilon)$ for $i=1,2, \ldots$.

Proposition 3.2. If $E$ is infinitely reproducible in $X$, then

$$
\Gamma_{\text {inf }}(X) \geq \Gamma(E) .
$$

Proof. Let $\operatorname{dim} E=n$. We claim that if $n$ divides $m$, then

$$
\Gamma_{m}(X) \geq \Gamma(E) .
$$

Indeed, let $\varepsilon>0$, let $E_{0}, E_{1}, E_{2}, \ldots \subset X$ and $P_{j}: Z \rightarrow E_{j}$ (here $Z=\overline{\left.E_{0} \oplus E_{1} \oplus E_{2} \oplus \ldots\right)}$ be projections so that, for $j=0,1,2, \ldots$,

$$
\left\|P_{j}\right\| \leq 1+\varepsilon, P_{j} P_{i}=0 \text { if } i \neq j \text {, and } d\left(E_{j}, E\right) \leq(1+\varepsilon) .
$$

For $j=0,1, \ldots$, let $\varphi_{1}^{j}, \ldots, \varphi_{n}^{j} \in B_{X^{*}}^{n}$ and $x_{1}^{j}, \ldots, x_{n}^{j} \in B_{E_{j}}^{n}$ be such that $G\left(\varphi_{1}^{j}, \ldots, \varphi_{n}^{j} ; x_{1}^{j}, \ldots, x_{n}^{j}\right)=G_{n}\left(E_{j}\right)$.

For $j=0,1, \ldots, n-1 ; i=1, \ldots, n$, let $x_{j n+i}=x_{i}^{j}$ and let $\varphi_{j n+i}$ be a Hahn-Banach extension to $X$ of $P_{j}^{*} \varphi_{i}^{j}$.

Write $m=n k, k \in \mathbb{N}$. Since $\left\langle\varphi_{j n+i}, x_{l n+s}\right\rangle=\delta_{j l}\left\langle\varphi_{i}^{j}, x_{s}^{j}\right\rangle$, we have

$$
\begin{array}{r}
G\left(\varphi_{1}, \ldots, \varphi_{m} ; x_{1}, \ldots, x_{m}\right)=\prod_{j=1}^{k} G\left(\varphi_{1}^{j}, \ldots, \varphi_{n}^{j} ; x_{1}^{j}, \ldots, x_{n}^{j}\right) \geq \\
\prod_{j=1}^{k} G\left(E_{j}\right) \geq \prod_{j=1}^{k}\left[d\left(E_{j}, E\right)^{-1} G(E)\right] \geq(1+\varepsilon)^{-k} G(E)^{k} .
\end{array}
$$

Hence $G_{m}(X) \geq(1+\varepsilon)^{-k} G(E)^{k}$, thus $G_{m}(X) \geq G(E)^{k}$ and (16) follows. The proposition follows from (16), because if $t=j n+i$, then $G_{t}(X) \geq G_{j n}(X) \geq \Gamma(E)^{j n}$, thus $\Gamma_{t}(X) \geq \Gamma(E)^{\frac{j n}{t}}$ and this goes to $\Gamma(E)$ when $t \rightarrow \infty$.

Recall that, given a property $(\mathrm{P})$, a space $X$ has property asymptotically- $P$, denoted (as. $\mathrm{P}$ ), if there is a sequence $Y_{n} \subset X$ of subspaces of finite codimension and a free ultrafilter on $\mathbb{N}$ such that the ultraproduct $Y=\prod Y_{n} / \mathcal{U}$ has the property $(\mathrm{P})$.

From propositions 3.1 and 3.2 we obtain the following

Corollary 3.1. $\Gamma$-spaces are asymptotically $W H$. 
It is, however, well known that if an ultraproduct is an as.WH space, then it is (isomorphic to) an assymptotically Hilbert space [16, chap. 14]. This proves the "asymptotically Hilbertian" part of Theorem 3.1. To get the "complementably" part of the theorem, we need the following proposition, which is an adaptation of a result due to Maurey and Pisier. For the convenience of the reader we reproduce a proof of it from [12].

Proposition 3.3. Let $X$ be a reflexive Banach space. For every subspace $Y \subset X$ of co-dimension $n$ and for every $\varepsilon>0$ with $m=(1+\varepsilon) n \in$ $\mathbb{N}$ there exists a projection $Q: X \rightarrow X$ whose co-rank is at most $m$ such that $Q X \subset Y$ and $\|Q\| \leq\left[G_{m+1}^{1 / m}(X)\right]^{\frac{1+\varepsilon}{\varepsilon}}$.

Proof. For $E \subset X, F \subset X^{*}$ let us denote

$$
G(E, F)=\sup \left\{|G(\varphi, x)|: \varphi \in B_{F}^{n}, x \in B_{E}^{n}\right\} .
$$

For $k=n, n+1, \ldots, m+1$ we shall define by induction $k$-dimenensional subspaces $E_{k}$ of $X$ and $F_{k}$ of $X^{*}$,

$$
E_{n} \subset E_{n+1} \subset \cdots \subset E_{m+1}, F_{n} \subset F_{n+1} \subset \cdots \subset F_{m+1}
$$

as well as a sequence of projections $P_{n}: X \rightarrow X$.

Let $F_{n}=Y^{\perp}$ and let $E_{n} \subset X$ be such that $\operatorname{dim} E_{n}=n$ and $G\left(E_{n}, F_{n}\right)=1$ (it exists, by the reflexivity and by $(7)$ ).

Let $m \geq k \geq n$, and assume that we have defined $E_{k} \subset X, F_{k} \subset$ $X^{*}$. Let $P_{k}: X \rightarrow X$ be the projection onto $E_{k}$ with $\operatorname{ker} P_{k}=$ $F_{k}^{\perp}$. Let $x_{i}^{*} \in B_{X^{*}}, x_{i} \in B_{X}, i=1, \ldots, k$ be such that $G\left(E_{k}, F_{k}\right)=$ $G\left(x_{1}^{*}, \ldots, x_{k}^{*} ; x_{1}, \ldots, x_{k}\right)$.

We define $x_{k+1} \in B_{X}$ and $x_{k+1}^{*} \in B_{X^{*}}$ so that

$$
\left\langle x_{k+1}^{*}, x_{k+1}-P_{k}\left(x_{k+1}\right)\right\rangle=\left\|I_{X}-P_{k}\right\| .
$$

Since $P_{k}\left(x_{k+1}\right) \in \operatorname{span}\left\{x_{1}, \ldots, x_{k}\right\}$, the determinant of the matrix $\left[\left\langle x_{i}^{*}, x_{j}\right\rangle\right]_{i, j=1}^{k+1}$ does not change if its last column (corresponding to $x_{k+1}$ ) is replaced by the vector $\left(0, \ldots, 0,\left\langle x_{k+1}^{*}, x_{k+1}-P_{k}\left(x_{k+1}\right)\right\rangle\right)$. We set $F_{k+1}=\operatorname{span}\left\{x_{1}^{*}, \ldots, x_{k+1}^{*}\right\}, E_{k+1}=\operatorname{span}\left\{x_{1}, \ldots, x_{k+1}\right\}$. Consequently, we have

$$
\begin{aligned}
& G\left(E_{k+1}, F_{k+1}\right) \geq \operatorname{det}\left[\left\langle x_{i}^{*}, x_{j}\right\rangle\right]_{i, j=1}^{k+1}= \\
& \left\langle x_{k+1}^{*}, x_{k+1}-P_{k}\left(x_{k+1}\right)\right\rangle G\left(E_{k}, F_{k}\right)=\left\|I_{X}-P_{k}\right\| G\left(E_{k}, F_{k}\right) .
\end{aligned}
$$

Since $G\left(E_{n}, F_{n}\right)=1$, we have for $m \geq k \geq n$

$$
G\left(E_{k+1}, F_{k+1}\right) \geq \prod_{j=n}^{k}\left\|I_{X}-P_{j}\right\| .
$$


For $k=m$, the right-hand side has $\varepsilon n$ elements, thus at least one of them is less than $G_{m+1}(X)^{\frac{1}{\varepsilon n}}$, say $\left\|I_{X}-P_{j}\right\| \leq G_{m+1}(X)^{\frac{1}{\varepsilon n}}=$ $\left[G_{m+1}^{1 / m}(X)\right]^{\frac{1+\varepsilon}{\varepsilon}}$. We set $Q=I_{X}-P_{j}$.

Remark 3.2. It is clear that very little happens if we drop the assumption of reflexivity: the assertion is true with $\|Q\|<\gamma$ where $\gamma$ is any number larger than $\left[G_{m+1}^{1 / m}(X)\right]^{\frac{1+\varepsilon}{\varepsilon}}$.

\section{4. $\Gamma$-SPACES ARE LIDSKII SPACES}

We shall apply Fredholm determinant theory, as presented in [16, chap. 15]. Let us recall some basic notions and facts.

For $n=1,2, \ldots$ and $x_{1}, x_{2}, \cdots \in X, x_{1}^{*}, x_{2}^{*}, \cdots \in X^{*}$ we set

$$
\alpha_{n}\left(x_{1}^{*} \otimes x_{1}, \ldots, x_{n}^{*} \otimes x_{n}\right)=\frac{1}{n !} \operatorname{det}\left(\left[\left\langle x_{j}^{*}, x_{i}\right\rangle\right]\right)
$$

and for $T_{1}, \ldots, T_{n} \in F(X)$ we define $\alpha_{n}\left(T_{1}, \ldots, T_{n}\right)$ by the $n$-linear extension.

Lemma 4.1. [16, Prop. 15.3.i]

$$
\alpha_{n}\left(T_{1}, \ldots, T_{n}\right) \leq \frac{G_{n}(X)}{n !}\left\|T_{1}\right\|_{\wedge} \ldots\left\|T_{n}\right\|_{\wedge} .
$$

Now, by continuity, we can extend $\alpha_{n}$ to any $T_{1}, \ldots, T_{n} \in N(X)$. For $T \in N(X)$ denote $\alpha_{n}(T)=\alpha_{n}(T, \ldots, T)$. Let us observe that if $T=\sum_{i=1}^{\infty} x_{i}^{*} \otimes x_{i}$ with $\sum\left\|x_{i}^{*}\right\|\left\|x_{i}\right\|<\infty$, then $\alpha_{1}(T)=\operatorname{tr} T\left(=\sum_{i=1}^{\infty} x_{i}^{*}\left(x_{i}\right)\right)$. This is so, because $\alpha_{1}$ is a continuous linear functional on $N(X)$ and $\alpha_{1}\left(x^{*} \otimes x\right)=x^{*}(x)$.

Since $G_{n}(X) \leq d_{n}(X)^{n} \leq n^{n / 2}$, we get for every $T \in N(X)$,

$$
\alpha_{n}(T) \leq \frac{n^{n / 2}}{n !}\|T\|_{\wedge}^{n} .
$$

Thus the series $\sum \alpha_{n}(T)$ converges absolutely for every $T \in N(X)$ and we set

$$
\operatorname{det}(I+T)=\sum_{n=0}^{\infty} \alpha_{n}(T) \text { with } \alpha_{0}(T)=1
$$

and for $z \in \mathbb{C}$ we define

$$
D_{T}(z)=\operatorname{det}(I+z T)=\sum_{n=0}^{\infty} \alpha_{n}(T) z^{n} .
$$

$D_{T}(z)$ is obviously an entire function for every $T \in N(X)$. It is well known that the zeros of $D_{T}$ are precisely the inverses of the non-zero eigenvalues of $T$. 
Let us observe that the order of the entire function $D_{T}$ is at most 2 . Indeed, by a basic formula (cf. [1, Theorem 2.2.2.]), if $f(z)=\sum \alpha_{n} z^{n}$, then the order of $f, \varrho(f)$ is equal to $\left[\lim \sup \frac{n \log n}{\log \left(\frac{1}{\left|\alpha_{n}\right|}\right)}\right]$. By (19) and by Stirling's formula,

$$
\begin{aligned}
\log \left(\frac{1}{\left|\alpha_{n}\right|}\right) & \geq \log \left(\frac{n !}{d_{n}(X)^{n}\|T\|_{\wedge}^{n}}\right) \geq \\
n\left(\log n-1-\log \|T\|_{\wedge}-\log d_{n}(X)\right) & \geq \frac{1}{2} n \log n-o(n \log n),
\end{aligned}
$$

thus $\varrho\left(D_{T}\right) \leq 2$.

Proposition 4.1. Assume that $d_{n}(X)=o\left(n^{\gamma}\right)$ with $\gamma<\frac{1}{2}$. If $T \in$ $N(X)$ and $T$ is quasi-nilpotent, then $D_{T}(z)=\exp (a z)$ for some $a \in \mathbb{C}$.

Proof. By $(20), \log \left(\frac{1}{\left|a_{n}\right|}\right) \geq(1-\gamma) n \log n-o(n \log n)$, thus $\varrho\left(D_{T}\right) \leq$ $\frac{1}{1-\gamma}<2$, hence $\varrho\left(D_{T}\right) \leq 1$.

$T$ being quasi-nilpotent, $D_{T}$ does not have any zeros, thus by the Hadamard factorization theorem [1, Theorem 2.7.1.], $D_{T}(z)$ must have the form $\exp (a z+b)$ with some $a, b \in \mathbb{C}$.

Since $D_{T}(0)=1$, we have $\exp b=1$.

Lemma 4.2. [16, Lemma 15.4] For every $T \in N(X)$ and every $\varepsilon>0$ there exists $C=C_{\varepsilon}(T)$ such that for every $n$,

$$
\left|\alpha_{n}(T)\right| \leq C \frac{G_{n}(X)}{n !} \varepsilon^{n} .
$$

Proof. Let $U \in F(X)$ be such that $\|T-U\|_{\wedge} \leq \frac{\varepsilon}{2}$. Set $V=T-U$. We have

$$
\alpha_{n}(T)=\sum_{j=0}^{n}\left(\begin{array}{l}
n \\
j
\end{array}\right) \alpha_{n}(U, \ldots, U, V, \ldots, V)
$$

( $U j$ times). Let $k=\operatorname{rk} U$, thus for $n \geq k$ we have

$$
\alpha_{n}(T)=\sum_{j=0}^{k}\left(\begin{array}{l}
n \\
j
\end{array}\right) \alpha_{n}(U, \ldots, U, V, \ldots, V) .
$$

By (19),

$$
\begin{array}{r}
\left|\alpha_{n}(U, \ldots, U, V, \ldots, V)\right| \leq \frac{G_{n}(X)}{n !}\|U\|_{\wedge}^{j}\|V\|_{\wedge}^{n-j} \\
\leq \frac{G_{n}(X)}{n !} \varepsilon^{n} 2^{-n} 2^{j} \varepsilon^{-j}\|U\|_{\wedge}^{j},
\end{array}
$$

thus (21) holds with $C=\max _{n} 2^{-n} \sum_{j=0}^{k}\left(\begin{array}{c}n \\ j\end{array}\right) 2^{j} \varepsilon^{-j}\|U\|_{\wedge}^{j}$. 
As a corollary we obtain

Theorem 4.1. If $X$ is a $\Gamma$-space, then $X$ is a weak Lidskii space; i.e., $X$ satisfies (WL).

Proof. Let $K<\infty$ be such that $G_{n}(X) \leq K^{n}$ for infinitely many $n$. We know that $X$ is asymptotically Hilbertian, thus $d_{n}(X)=o\left(n^{\gamma}\right)$ for every $\gamma>0$, by Proposition 2.1. Let $T \in N(X)$ be quasi-nilpotent. By Proposition 4.1, $D_{T}(z)=\exp (a z)$ for some $a \in \mathbb{C}$, hence $\left|\alpha_{n}(T)\right|=$ $\frac{|a|^{n}}{n !}$. Fix an $\varepsilon>0$. By Lemma $3,|a| \leq C_{\varepsilon}^{\frac{1}{n}} K \varepsilon$ for infinitely many $n$, thus $a=0$. This shows that formula (L) holds for all quasi-nilpotent operators in $N(X)$. By [3], this implies that $X$ is a weak Lidskii space.

Combining Theorem 4.1 with the result from [3] that (WL) implies (L) we get

Corollary 4.1. If $X$ is a $\Gamma$-space, then $X$ is a Lidskii space.

Since [3] has yet to be written, we sketch a proof of

Theorem 4.2. (Figiel-Johnson) If $X$ satisfies (WL), then $X$ satisfies (L).

Proof. The main tool is Ringrose's [17] structure theory for compact operators. Let $T$ be a compact operator on a complex Banach space $X$ and let $\mathcal{N}$ be a maximal nest of closed subspaces of $X$ that are invariant for $T$. Given $N \in \mathcal{N}$, let $N^{-}$be the closed linear span of all $M$ in $\mathcal{N}$ that are properly contained in $N$. Ringrose observes that either $\mathcal{N}^{-}=N$ or $N^{-}$has codimension one in $N$. In the latter case, there is an eigenvalue $\lambda_{N}$ of $T$ so that for every $x \in N \sim N^{-}$we have $T x=\lambda_{n} x+y_{x}$ with $y_{x} \in N^{-}$. The collection $\mathcal{N}^{\prime}:=\left\{\lambda_{N}: \operatorname{dim} N / N^{-}=1\right\}$ exhausts the eigenvalues of $T$ repeated according to multiplicity, and so the collection $\mathcal{N}^{\prime}$ is countable.

Suppose now that $\sum_{N \in \mathcal{N}^{\prime}}\left|\lambda_{N}\right|<\infty$. For $N \in \mathcal{N}$ pick $x_{N} \in N$ of norm one so that the distance of $x_{N}$ to $N^{-}$is close to one. choose a functional $x_{N}^{*} \in\left(N^{-}\right)^{\perp}$ with norm close to one so that $x_{N}^{*}\left(x_{N}\right)=1$. Then the linear operator $S:=\sum_{N \in \mathcal{N}^{\prime}} \lambda_{n} x_{N}^{*} \otimes x_{N}$ is nuclear and every $N \in \mathcal{N}$ is an invariant subspace for $S$. Consequently, $\mathcal{N}$ is a (necessarily maximal) nest of invariant subspaces for the compact operator $T-S$. By construction, for every $N \in \mathcal{N}^{\prime}$ we have that $T N \subset N^{-}$, which is to say that $T-S$ is quasi-nilpotent, and of course nuclear if $T$ is nuclear. 


\section{EXAMPles OF $\Gamma$-SPACES}

We shall say that $X$ is asymptotically Hilbertian of polynomial growth if there is a constant $\lambda$ such that there are subspaces $Y_{1}, Y_{2}, \cdots \subset X$ with $\operatorname{dim} X / Y_{n}=O\left(n^{\lambda}\right)$ and $\liminf d_{n}\left(Y_{n}\right)<\infty$.

Proposition 5.1. In the above definition, "there is $\lambda$ " implies "for every $\lambda>0$ ".

Proof. Let $n_{1}<n_{2}<\ldots$ be a sequence such that for every $j, d_{n_{j}}\left(Y_{n_{j}}\right) \leq$ $d<\infty$ and $\operatorname{dim} X / Y_{n_{j}} \leq C n_{j}^{\lambda}$. Let $m_{j}=n_{j}^{\gamma}$ and let $Z_{m_{j}}=Y_{n_{j}}$ for $j=1,2, \ldots$ By $(14)$, we have $d_{m_{j}}\left(Z_{m_{j}}\right) \leq d^{2 \gamma}$ whereas $\operatorname{dim} X / Z_{m_{j}} \leq$ $C m_{j}^{\frac{\lambda}{\gamma}}$. Taking $\gamma$ sufficiently large, we get $\frac{\lambda}{\gamma}$ as small as we wish.

Theorem 5.1. If $X$ is complementably asymptotically Hilbertian of polynomial growth, then $X$ is a $\Gamma$-space.

Theorem 5.1 follows from

Lemma 5.1. Let $P: X \rightarrow X$ be a rank $k$ projection and set $Y=\operatorname{ker} P$. Denote $K=\max \left(\|P\|,\left\|I_{X}-P\right\|\right)$. Then

$$
G_{n}(X) \leq K^{2 n} n^{2 k} d_{n}(Y)^{n} ; \text { i.e. } \Gamma_{n}(X) \leq K^{2} n^{\frac{2 k}{n}} d_{n}(Y) .
$$

Proof. For $\varphi \in X^{*}, x \in X$ denote

$$
\varphi^{1}=P^{*} \varphi, \varphi^{0}=\left(I_{X}-P^{*}\right) \varphi, x^{1}=P x, x^{0}=\left(I_{X}-P\right) x .
$$

For $\varepsilon \in\{0,1\}^{n}$ let $|\varepsilon|=\sum_{j=1}^{n} \varepsilon_{j}$.

For $\varepsilon, \eta \in\{0,1\}^{n}, \varphi \in X^{* n}, x \in X^{n}$ denote $G_{\varepsilon, \eta}(\varphi, x)=\operatorname{det}\left[\left\langle\varphi_{i}^{\eta(i)}, x_{j}^{\varepsilon(j)}\right\rangle\right]$. By the $2 n$-linearity of $G(\varphi, x)$, we have

$$
G(\varphi, x)=\sum_{\varepsilon, \eta \in\{0,1\}^{n}} G_{\varepsilon, \eta}(\varphi, x) .
$$

Clearly $\left\langle\varphi_{i}^{1}, x_{j}^{0}\right\rangle=\left\langle\varphi_{i}^{0}, x_{j}^{1}\right\rangle=0$ for all $i, j$, therefore $G_{\varepsilon, \eta}(\varphi, x)=0$ unless $|\varepsilon|=|\eta|$. For $A, B \subset\{1, \ldots, n\}$ such that $|A|=|B|$, let $G_{A, B}^{1}(\varphi, x)$ be the minor of the matrix $\left[\left\langle\varphi_{i}^{1}, x_{j}^{1}\right\rangle\right]_{i, j=1}^{n}$, corresponding to the rows in $A$ and to the columns in $B$ and let $G_{A, B}^{0}(\varphi, x)$ be the minor of the matrix $\left[\left\langle\varphi_{i}^{0}, x_{j}^{0}\right\rangle\right]_{i, j=1}^{n}$, corresponding to the rows in $A^{c}$ and to the columns in $B^{c}$. We see that

$$
G_{\varepsilon, \eta}(\varphi, x)=\sigma(A, B) G_{A, B}^{1}(\varphi, x) G_{A, B}^{0}(\varphi, x),
$$


where $\sigma(A, B)= \pm 1$ and $A=\{i: \varepsilon(i)=1\}, B=\{j: \varphi(j)=1\}$. Since $\operatorname{dim} P X=k$, we have $G_{A, B}^{1}=0$ for $|A|=|B|>k$, thus

$$
G(\varphi, x)=\sum_{A, B \subset\{1, \ldots, n\},|A|=|B| \leq k} \sigma(A, B) G_{A, B}^{1}(\varphi, x) G_{A, B}^{0}(\varphi, x) .
$$

Let us observe that for $|A|=|B|=j$ we have

$$
\left|G_{A, B}^{1}(\varphi, x)\right| \leq\|P\|^{2 j} G_{j}(P X),\left|G_{A, B}^{0}(\varphi, x)\right| \leq\left\|I_{X}-P\right\|^{2(n-j)} G_{n-j}(Y),
$$

therefore

$$
|G(\varphi, x)| \leq K^{2 n} \sum_{j=0}^{k}\left(\begin{array}{l}
n \\
j
\end{array}\right)^{2} G_{j}(P X) G_{n-j}(Y) .
$$

By Lemma 2.1 we have

$$
G_{j}(P X) \leq j !^{1 / 2} \text { and } G_{n-j}(Y) \leq G_{n}(Y)
$$

thus

$$
|G(\varphi, x)| \leq K^{2 n} n^{2 k} G_{n}(Y) \leq K^{2 n} n^{2 k} d_{n}(Y)^{n},
$$

since $G_{n}(Y) \leq d_{n}(Y)^{n}$.

Proof of Theorem 5.1. By the definition, there are $\beta, K, C<\infty$ and $Y_{n}$ for $n=1,2,3, \ldots$ so that $q_{n}=\operatorname{dim} X / Y_{n}<\infty$ and (i),(ii),(iii) below are satisfied:

(i) $d_{n}\left(Y_{n}\right) \leq \beta$,

(ii) $\liminf q_{n} \log n / n<\infty$,

(iii) there are projections $P_{n}: X \rightarrow Y_{n}$ with $\left\|P_{n}\right\|,\left\|I_{X}-P_{n}\right\| \leq K$

((ii) follows from Proposition 5.1). By Lemma 5.1, $\Gamma_{q_{n}}(X) \leq K^{2} \beta e^{\frac{3}{2} C}$.

The primary example are spaces of the form $X=\left(\sum_{n=1}^{\infty} \oplus \ell_{p_{n}}^{k_{n}}\right)_{2}$.

Denote $\delta_{n}=\left|p_{n}-2\right|$. We have for $n \leq m, d_{n}\left(\ell_{p}^{m}\right)=n^{\left|\frac{1}{2}-\frac{1}{p}\right|}$, thus $\frac{1}{6}|p-2| \leq d_{n}\left(\ell_{p}^{m}\right) \leq \frac{1}{2}|p-2|$ for $1 \leq p \leq 3, n \leq m$. Therefore

$\left.{ }^{*}\right) X$ is not isomorphic to a Hilbert space iff $\sup k_{m}^{\delta_{m}}=\infty$.

Let us make two ad hoc assumptions:

$$
\delta_{n} \searrow 0, k_{n} \geq 2 k_{n-1} \text { for every } n \text {. }
$$

Set $Z_{m}=\{0\} \oplus \cdots \oplus\{0\} \oplus\left(\sum_{n=m+1}^{\infty} \oplus \ell_{p_{n}}^{k_{n}}\right)_{2}$. Then

$$
d_{n}\left(Z_{m}\right) \leq d_{n}\left(\ell_{p_{m+1}}\right)=n^{\delta_{m+1}}, \operatorname{codim}\left(Z_{m}\right)=k_{1}+\cdots+k_{m} \leq 2 k_{m} .
$$

The next two lemmas follow now from elementary computations: 
Lemma 5.2. $X$ is asymptotically Hilbertian of polynomial growth provided the sequence $\left\{k_{m}^{\delta_{m+1}}\right\}$ is bounded.

Together with $(*)$ this gives

Lemma 5.3. If $\frac{\log k_{m+1}}{\log k_{m}} \rightarrow \infty$ and $\delta_{m+1}=\frac{C}{\log k_{m}}$, then $X$ is complementably asymptotically Hilbertian of polynomial growth and not isomorphic to a Hilbert space.

An immediate consequence of the preceding lemma and the constructions and results in [2] we get

Theorem 5.2. The direct sum of two Lidskii spaces need not be a Lidskii space.

In fact, if $p_{n} \rightarrow 2$ and $k_{n} \rightarrow \infty$ and $X=\left(\sum_{n=1}^{\infty} \oplus \ell_{p_{n}}^{k_{n}}\right)_{2}$, we can get a partition $N=N_{1} \cup N_{2}$ so that both $X_{1}:=\left(\sum_{n \in X_{1}} \oplus \ell_{p_{n}}^{k_{n}}\right)_{2}$ and $X_{2}:=\left(\sum_{n \in X_{2}} \oplus \ell_{p_{n}}^{k_{n}}\right)_{2}$ are complementably asymptotically Hilbertian of polynomial growth, while $X=X_{1} \oplus X_{2}$ can even fail to be HAPpy if the rates $p_{n} \rightarrow 2$ and $k_{n} \rightarrow \infty$ are chosen appropriately-see [2].

\section{Open questions}

Question 1. Suppose $G_{n}(X)$ is bounded or $G_{n}(X)^{\frac{1}{n}} \rightarrow 1$. Does it follow that $X$ is (isomorphic to) a Hilbert space?

Question 2. If $X$ is isomorphic to a Hilbert space, does it follow that $G_{n}(X)$ is bounded or that $G_{n}(X)^{\frac{1}{n}} \rightarrow 1$ ?

Question 3. Is (HAP) equivalent to (L)?

Question 4. Is every asymptotically Hilbertian space CAH?

Question 5. Suppose that $d_{n}(X)$ goes to infinity sufficiently slowly. Must $X$ be a Lidskii space?

\section{REFERENCES}

[1] R. Boas, Entire Functions, New York : Academic Press, 1954

[2] P. G. Casazza, C. L. Garcia, and W. B. Johnson, An example of an asymptotically Hilbertian space which fails the approximation property, Proc. AMS 129 (2001), 3017-3023.

[3] T. Figiel and W. B. Johnson, in preparation.

[4] A. Grothendieck, Produits Tensoriels Topologiques et Espaces Nucleaires, Memoirs AMS 16 (1955).

[5] A. Grothendieck, La théorie de Fredholm, Bull. Soc. Math. France 84 (1956), 319-384. 
[6] W. B. Johnson, Banach spaces all of whose subspaces have the approximation property, (Proc. Sem., Ges. Math. Datenverarb., Bonn, 1979), North-Holland, Amsterdam-New York, (1980), 15-26.

[7] W. B. Johnson, H. König, B. Maurey, J. R. Retherford, Eigenvalues of $p$ summing and $l_{p}$-type operators in Banach spaces, J. Funct. Anal. 32 (1979), $353-380$.

[8] W. B. Johnson and A. Szankowski, Hereditary approximation property, Annals of Math. 176 (2012), 1987-2001.

[9] V. Lidskii, Non-self adjoint operators with a trace, Dokl.Akad.Nauk SSSR 125 (1959), 485-487.

[10] J. Lindenstrauss and L. Tzafriri, On the complemented subspaces problem, Israel J. Math. 9 (1971), 263-269.

[11] J. Lindenstrauss and L. Tzafriri, Classical Banach spaces I, Sequence spaces, Springer, Berlin 1977.

[12] V. Mascioni, On Banach spaces isomorphic to their duals, Houston J. Math. 19 (1993), 27-38

[13] V. Milman and G. Schechtman, Asymptotic theory of finite dimensional normed spaces. Springer-Verlag, Lecture Notes in Mathematics 1200, (1986).

[14] G. Pisier, Martingales with values in uniformly convex spaces, Israel J. Math. 20 (1975), 326-350.

[15] G. Pisier, Weak Hilbert spaces, Proc. London Math. Soc. (3) 56 (1988), no. 3, 547-579.

[16] G. Pisier, The volume of convex bodies and Banach space geometry, Cambridge Tracts in Math. 94 (1989).

[17] J. R. Ringrose, Super-diagonal forms for compact linear operators, Proc. London Math. Soc. (3), 12 (1962), 367-384.

[18] N. Tomczak-Jaegermann, Banach-Mazur distances and finite-dimensional operator ideals, Longman Scientific and Technical, 1989.

Department of Mathematics, Texas A\&M University, College StaTION, TX 77843-3368 U.S.A

E-mail address: johnson@math.tamu.edu

Institute of Mathematics, The Hebrew University , Jerusalem 91904 ISRAEL

E-mail address: tomek@math.huji.ac.il 